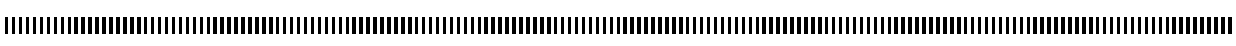

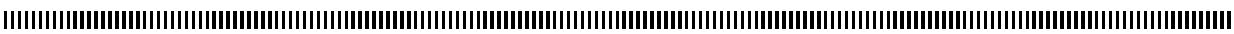

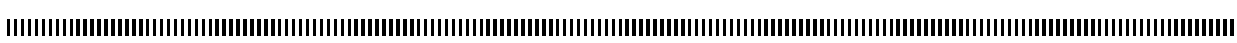

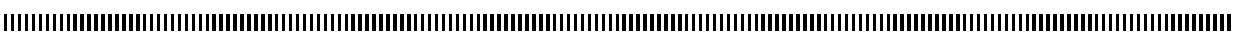

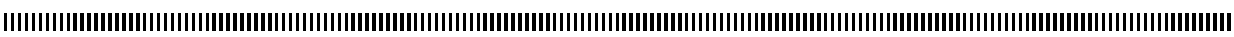

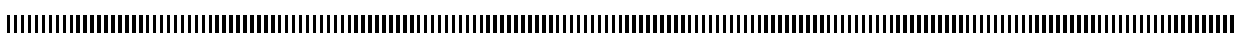

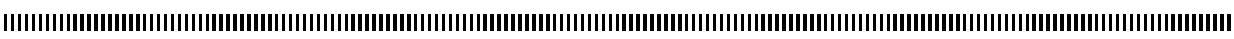

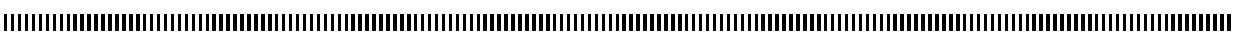

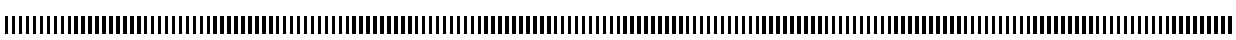

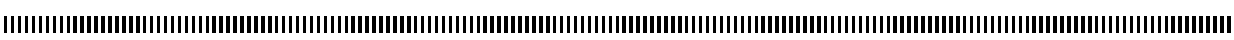

\title{
A stochastic modelling of phytoplankton aggregation
}

\author{
Nadjia El Saadi * — Ovide Arino** \\ * GEODES-IRD \\ 32 avenue Henri Varagnat \\ F-93143 Bondy Cedex \\ France \\ elsaadi@bondy.ird.fr; enadjia@yahoo.fr \\ *** Deceased on 29 Septembre 2003
}

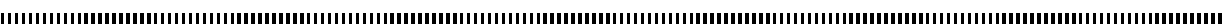

RÉSUMÉ. L' objectif de ce travail est de fournir un modèle mathématique stochastique qui décrit l'agrégation chez le phytoplancton, à partir de la modélisation d'un système de taille grande mais finie de cellules de phytoplancton sujettes à une dispersion aléatoire, des interactions spatiales qui donnent aux mouvements des cellules une certaine dépendance et un branchement (division cellulaire ou mort). Nous présentons le passage de la description microscopique à une description macroscopique, lorsque le nombre de cellules devient très grand (grandes populations de phytoplancton). La limite du système est une extension du superprocessus de Dawson-Watanabe : c'est un superprocessus avec interactions qui peut être décrit par une équation aux dérivées partielles stochastique non linéaire.

ABSTRACT. The aim of this work is to provide a stochastic mathematical model of aggregation in phytoplankton, from the point of view of modelling a system of a large but finite number of phytoplankton cells that are subject to random dispersal, mutual interactions allowing the cell motions some dependance and branching (cell division or death). We present the passage from the "microscopic" description to the "macroscopic" one, when the initial number of cells tends to infinity (large phytoplankton populations). The limit of the system is an extension of the Dawson-Watanabe superprocess: it is a superprocess with spatial interactions which can be described by a nonlinear stochastic partial differential equation.

MOTS-CLÉS : Agrégation du phytoplancton, Modèle Lagrangien, Processus de branchement diffusion interactif, Problème de martingales, Convergence faible, Superprocessus de Dawson-Watanabe, Equation aux dérivées partielles stochastique.

KEYWORDS : Phytoplankton aggregation, Lagrangian model, Interacting branching diffusion process, Martingale problem, Weak convergence, Dawson-Watanabe superprocess, Stochastic partial differential equation

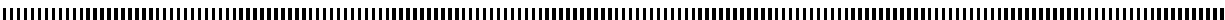

Volume 5 - 2006, pages 80-94 - ARIMA

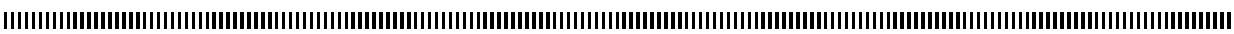

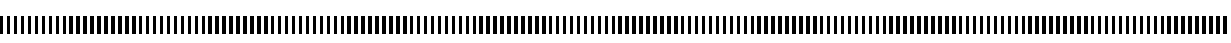
| | | |

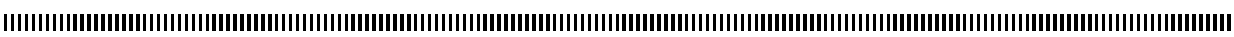
| ЧЕНแЕ 


\section{Introduction}

Phytoplankon play a key role in the marine ecology. These microscopic single-celled organisms have the ability of forming large aggregates which provide food for a tremendous variety of marine animals, including zooplankton (microscopic animals), bivalve molluscan shellfish (mussels, oysters, scallops and clams), and small fish (such as anchovies and sardines). These animals, in turn, provide food for other animals, including crabs, fish, marine birds, marine mammals and humans. Thus, spatial and temporal patterns of phytoplankton are very important to fisheries and the mathematical modelling of phytoplankton dynamics is a very interesting subject both for biologists and mathematicians.

Despite extensive field evidence for aggregate formation ([2],[14],[19]), the factors regulating aggregation are still unclear. Coagulation theory has more recently been applied to describe aggregation of marine aggregates and specifically phytoplankton aggregation. According to coagulation theory, aggregation of phytoplankton results from the repetitive collision of cells and their subsequent attachment to form larger aggregates. Brownian motion, fluid shear may all cause collision. Although some laboratory experiments, mesocosm experiments and field observations have demonstrated that coagulation theory at times provides an accurate description of phytoplankton aggregate formation (see [18]), the factors most critical to predicting aggregation in nature using coagulation theory remain unclear ([3],[6]).

In contrast with the coagulation theory which generally bases on pure physical processes, many studies of marine aggregates at small scales emphasize biological mechanisms for the phytoplankton aggregate formation. Indeed, some planktonic species (algae, bacteria, dinoflagellates) have chemosensory abilities that would be useless if the ocean was chemically homogeneous ( [13],[26],[27]). Dinoflagellates and more generally motile algae are known to leak organic matter such as amino-acids and sugar into water [21] and this leakage creates a zone around individual cells called the " phycosphere", where extracellular products exist in enhanced concentrations over background [4]. The chemosensory responses allow dinoflagellates and algae that are present in a suitable neighborhood to find the leaky cells and to stay near them forming aggregates. Experiments studies on the chemosensory capabilities in dinoflagellates and motile algae ([13],[27]) show that these organisms are attracted to many varieties of amino-acids, organic compounds and sugar but it has been reported that high concentrations of these products inhibit the chemosensory behavior.

This work addresses the issue of modelling phytoplankton aggregation. Our modelling strategy is to start with a microscopic Lagrangian description of the evolution of $N$ phytoplankton cells and then we pass to a continuous limit describing the spatial distribution of the population. The Lagrangian model provides an explanation of the aggregation behavior in phytoplankton cells in terms of attraction mechanisms among cells due to the chemosensory behavior, random branching (cell division and natural death), in addition to individual random dispersals described by independent Brownian motions. The advan- 
tage of this approach is to catch the main characteristics of the individual dynamics at small scales that are responsible at larger scale for the formation of aggregating patterns.

In [24], the authors have investigated the modelling of spatially structured populations with fixed sizes. Here in this paper, we add branching mechanisms for our interacting particle system, that is particles can divide into two or die. So, our model can be considered as a generalization of the class of models reviewed in [24]. We do not consider other processes for the planktonic particles such us growth, gravitational sinking or grazing by higher animals. We stress on the fact that, owing to the branching, the technique proposed in [24] for the derivation of the continuum model fails in our situation. So, to derive the limit, we use the approach in [22] based on martingale properties of a sequence of approximating interacting branching diffusion processes. The limit obtained is a "superprocess with spatial interactions" which is an extension of the Dawson-Watanabe superprocess.

The paper is organized as follows : in the next section, we describe the Lagrangian aggregation model of phytoplankton cells. In section 3 , we present the interacting branching-diffusion process to describe the spatial and temporal distribution of phytoplankton cells. We set the martingale problem associated to this process and characterize the latter as the unique solution to this martingale problem.

Section 4 is devoted to the limit of the system when the initial number of particles tends to infinity. Using the "Feller rescaling", we prove the weak convergence of the rescaled interacting branching-diffusion process to a "superprocess with interactions" and state the nonlinear stochastic partial differential equation satisfied by this superprocess. At the end of this section, we derive the heuristic nonlinear stochastic partial differential equation that should be satisfied by the density (if it exists) of the limiting process.

Finally, some remarks about notations and hypotheses in this paper :

$-C_{b}^{2}(\mathbb{R})$ is the space of bounded functions of class $C^{2}$ on $\mathbb{R}$, endowed with the supremum norm $\|\cdot\|_{\infty}$.

$-M_{F}(\mathbb{R})$ is the space of non negative finite measures on $\mathbb{R}$, endowed with the weak topology and $\langle$,$\rangle is the duality bracket. That is, a sequence of measures \left\{\mu_{n}\right\}$ in $M_{F}(\mathbb{R})$ converges (weakly) to the measure $\mu$ as $n \rightarrow \infty$ if, for each $\psi \in C_{b}(\mathbb{R}),\left\langle\mu_{n}, \psi\right\rangle \rightarrow\langle\mu, \psi\rangle$ as $n \rightarrow \infty$.

- For any metric space $S$, we denote by $D([0, \infty[, S)$ the space of càdlàg (right continuous with left limits) mappings from $[0, \infty[$ to $S$.

$-\mathcal{B}(\mathbb{R})$ is the $\sigma$-field of Borel sets of $\mathbb{R}$.

- When taking the expectation of a function of a Markov process or the distribution of a process, we use a subscript to specify the initial value of the process.

\section{Lagrangian aggregation model}

Let us consider $N(N \in \mathbb{N})$ phytoplankton cells, each of them having at some time a certain position in the space $\mathbb{R}$. Here, $\mathbb{R}$ represents the vertical axis oriented downward 
from the surface to the seabed. The $i^{t h}$ cell in the system is described by its position $X_{i}$ and its mass $m$.

The positions of the $N$ cells in the system evolve according to :

$$
d X_{i}(t)=\sum_{j=1, j \neq i}^{N} K\left(X_{i}(t), X_{j}(t)\right) d t+\sqrt{2 D} d B_{i}(t) \quad i=1, \ldots, N,
$$

where $B_{i}(t), i=1, \ldots, N$ are independent $\mathbb{R}$-valued Brownian motions. The dispersion term in (1) expresses the vertical diffusion of phytoplankton cells in water, which is similar to molecular diffusion ([5],[15]) and $D$ is the coefficient of diffusion. The drift term indicates the attractive force exerted on the $i^{t h}$ cell due to the interaction of this cell with all others in the system.

To describe interactions between phytoplankton cells, we propose the ideas involving non-uniformity of the concentration fields around organisms and consider planktonic species having chemosensory abilities (dinoflagellates, motile algae) and hence some knowledge about their neighbors. However, each cell has a limited knowledge of the spatial distribution of its neighbors because it is sensory limited ([5],[16],[17])).

So, to model interactions between phytoplankton cells, we may take into account these biological considerations :

1) A cell in position $x$ interacts with a cell in position $y$, if the distance $|x-y|$ is between $r_{0}$ and $r_{\max }\left(r_{0}\right.$ and $r_{\max }$ belong to $\left.\mathbb{R}_{+}^{*}, r_{0} \ll r_{\max }\right)$.

2) If the distance $|x-y|>r_{\max }$, the two cells do not interact because they cannot perceive differences in concentration at a distance over $r_{\max }$.

3 ) If the distance $|x-y|<r_{0}$, the cell in $x$ stops to be attracted to the cell in position $y$ because of the largest concentration in the closest vicinity of $y$ [13].

Therefore, we define pair interaction forces as follows :

the interaction between two cells at positions $x$ and $y$ depends on the distance between the two cells and is determined by :

$$
K(x, y)=m F_{a}(x-y)
$$

where $F_{a}$ is an attractive force defined by :

$$
F_{a}(z)=\left\{\begin{array}{cl}
-|z|^{2}+\left(r_{0}+r_{\max }\right)|z|-r_{0} r_{\max } & \text { if } \quad r_{0}<|z|<r_{\max } \\
0 & \text { otherwise }
\end{array} .\right.
$$

The sum $\sum_{j=1, j \neq i}^{N} K\left(X_{i}(t), X_{j}(t)\right)$ in (1) represents the effect of all cells on the $i^{t h}$ cell located in $X_{i}(t)$ at time $t$.

The cells also branch. In phytoplankton, the most common mean of reproduction is asexual cell division (mitosis). This process splits the cell into two identical copies. Therefore, we describe the dynamics of the system as follows : 
a phytoplankton cell performs a motion in some region in $\mathbb{R}$, according equation (1). In a time interval $[t, t+h]$, the cell has a probability $\mu h+o(h)$ of branching : it either splits into 2 identical cells or it dies out with probability $\frac{1}{2}$ each. If it splits, the 2 new cells begin their life at the branching point. They continue their motion following equation (1) until the time themselves branch and so on.

We suppose that the initial configuration of the system is described by a measure $\nu \in M_{F}(\mathbb{R})$ and that the initial number of cells, $N$, is large but finite.

\section{Spatial and temporal distribution of a finite system of phytoplankton cells}

The collective behavior of phytoplankton cells at time $t$ is described by the empirical random measure process $\left\{\eta_{t}\right\}$ (the interacting branching-diffusion process) on $\mathbb{R}$ :

$$
\eta_{t}=\sum_{i=1}^{N(t)} \delta_{X_{i}(t)}
$$

where $\delta_{X_{i}(t)}$ is the Dirac measure at the location $X_{i}(t) \in \mathbb{R}$ of the cell $i$ at time $t$ and $N(t)$ is the total number of alive cells in the system at time $t$. For every $B \in \mathcal{B}(\mathbb{R}), \eta_{t}(B)$ counts the number of alive cells present in $B$ at time $t$.

In [8], we show that the infinitesimal generator of the interacting branching-diffusion process $\left\{\eta_{t}\right\}$ which we denote by $£$, can be written as :

$$
£ F_{\psi}(\varepsilon)=£_{d} F_{\psi}(\varepsilon)+£_{b} F_{\psi}(\varepsilon)
$$

with

$$
£_{d} F_{\psi}(\varepsilon)=\left\langle\varepsilon, \frac{D \psi^{\prime \prime}+m\left(F_{a} * \varepsilon\right) \psi^{\prime}}{\psi}\right\rangle \exp \langle\varepsilon, \log \psi\rangle
$$

and

$$
£_{b} F_{\psi}(\varepsilon)=\left\langle\varepsilon, \frac{\mu[\Phi(\psi)-\psi]}{\psi}\right\rangle \exp \langle\varepsilon, \log \psi\rangle
$$

where $\Phi$ is the generating function of the offspring distribution $\left(\Phi(s)=\frac{1}{2}+\frac{1}{2} s^{2}\right)$, $F_{\psi}(\varepsilon)=\exp \langle\varepsilon, \log \psi\rangle$ with $\varepsilon \in M_{F}(\mathbb{R}), \psi \in C_{b}^{2}(\mathbb{R})$ and $\|\psi\|_{\infty} \leq 1$. The term $£_{d}$ is relative to the spatial motion dynamics and $£_{b}$ is induced by the branching dynamics. The domain of $£$ is all such functions $F_{\psi}$ for which $£ F_{\psi}$ is bounded.

Our approach is to characterize the distribution of the process $\left\{\eta_{t}\right\}$ as a solution to certain martingale problem. Martingale characterizations are very useful techniques which make good mathematical sense in situations where for example stochastic partial differential equation technology breaks down. Furthermore, they are well suited to weak convergence methods. We recall : 


\section{Definition 1 (Martingale Problem)}

We say that a stochastic process $\{\epsilon(t)\}_{t}$, or equivalently its distribution $P_{\pi}$, solves the $(£, \pi)$ martingale problem (where $\left.\pi \in M_{F}(\mathbb{R})\right)$ if :

$$
P_{\pi}[\epsilon(0)=\pi]=1
$$

and

$$
F(\epsilon(t))-F(\epsilon(0))-\int_{0}^{t} £ F(\epsilon(s)) d s
$$

is a $P_{\pi}$-martingale for any function $F \in D(£)$.

Now, we can state the main result on the characterization of the process $\left\{\eta_{t}\right\}$ :

Theorem 1 (Martingale characterization)

1) The process $\left\{\eta_{t}\right\}_{t}$ with initial measure $\nu$ is the unique solution of the martingale problem $(£, \nu)$ : that is, the law $P_{\nu}$ of $\left\{\eta_{t}\right\}$ satisfies :

$\forall \psi>0, \psi \in C_{b}^{2}(\mathbb{R})$ and $\|\psi\|_{\infty} \leq 1$

$$
F_{\psi}\left(\eta_{t}\right)-F_{\psi}(\nu)-\int_{0}^{t} £ F_{\psi}\left(\eta_{s}\right) d s
$$

is a $P_{\nu}$-martingale.

2) $\forall \varphi>0, \varphi \in C_{b}^{2}(\mathbb{R})$

$$
M_{t}(\varphi) \triangleq\left\langle\eta_{t}, \varphi\right\rangle-\left\langle\eta_{0}, \varphi\right\rangle-\int_{0}^{t}\left\langle\eta_{s}, D \varphi^{\prime \prime}+m\left(F_{a} * \varepsilon\right) \varphi^{\prime}\right\rangle d s
$$

is a $P_{\nu}$-martingale. Moreover, the quadratic variation of $M_{t}(\varphi)$ is

$$
\langle M(\varphi)\rangle_{t}=\int_{0}^{t}\left\langle\eta_{s}, 2 D\left(\varphi^{\prime}\right)^{2}+2 \mu \frac{\Phi(\exp (-\varphi))-\exp (-\varphi)}{\exp (-\varphi)}\right\rangle d s .
$$

\section{Proof.}

1) Since $\left\{\eta_{t}\right\}_{t}$ is a Markov process, it is the unique solution of the martingale problem for its generator $£$ ( See [11], chap 4).

2) Let $\varphi \in C_{b}^{2}(\mathbb{R})>0$ and take $\psi=\exp (-\theta \varphi)$ for some real constant $\theta \geq 0$. Since $\left(\eta_{t}\right)_{t}$ is a solution of the martingale problem $(£, \nu)$, it is easy to check that :

$$
E_{\nu}\left[F_{\psi}\left(\eta_{t+u}\right)-F_{\psi}\left(\eta_{t}\right)-\int_{t}^{t+u} £ F_{\psi}\left(\eta_{s}\right) d s / t\right]=0
$$


with

$$
F_{\psi}\left(\eta_{t}\right)=\exp \left(\left\langle\eta_{t}, \log \psi\right\rangle\right)=\exp \left(-\left\langle\eta_{t}, \theta \varphi\right\rangle\right)
$$

Using that

$$
\begin{aligned}
£ F_{\psi}\left(\eta_{s}\right) & =\left\langle\eta_{s},-D \theta \varphi^{\prime \prime}+D \theta^{2}\left(\varphi^{\prime}\right)^{2}-m \theta\left(F_{a} * \eta_{s}\right) \varphi^{\prime}\right. \\
& \left.+\mu \frac{\Phi(\exp (-\varphi))-\exp (-\varphi)}{\exp (-\varphi)}\right\rangle \exp \left(-\left\langle\eta_{s}, \theta \varphi\right\rangle\right),
\end{aligned}
$$

differentiating with respect to $\theta$ and setting $\theta=0$, we obtain :

$$
E_{\nu}\left[\left\langle\eta_{t+u}, \varphi\right\rangle-\left\langle\eta_{t}, \varphi\right\rangle-\int_{t}^{t+u}\left\langle\eta_{s}, D \varphi^{\prime \prime}+m\left(F_{a} * \eta_{s}\right) \varphi^{\prime}\right\rangle d s / t\right]=0,
$$

that is (2) is a $P_{\nu}$-martingale and $\left\langle\eta_{t}, \varphi\right\rangle$ is a semimartingale.

Applying Itô's formula, we have that $\exp \left(-\left\langle\eta_{t}, \varphi\right\rangle\right)$ is a semimartingale and

$$
\begin{gathered}
\exp \left(-\left\langle\eta_{t}, \varphi\right\rangle\right)-\exp (-\langle\nu, \varphi\rangle)+\int_{0}^{t} \exp \left(-\left\langle\eta_{s}, \varphi\right\rangle\right)\left\langle\eta_{s}, D \varphi^{\prime \prime}+m\left(F_{a} * \eta_{s}\right) \varphi^{\prime}\right\rangle d s \\
-\frac{1}{2} \int_{0}^{t} \exp \left(-\left\langle\eta_{s}, \varphi\right\rangle\right) d\langle M(\varphi)\rangle_{s}
\end{gathered}
$$

is a $P_{\nu}$-martingale.

On an other hand,

$$
\begin{gathered}
\exp \left(-\left\langle\eta_{t}, \varphi\right\rangle\right)-\exp (-\langle\nu, \varphi\rangle)+\int_{0}^{t} \exp \left(-\left\langle\eta_{s}, \varphi\right\rangle\right)\left\langle\eta_{s}, D \varphi^{\prime \prime}-D\left(\varphi^{\prime}\right)^{2}\right. \\
\left.+m\left(F_{a} * \eta_{s}\right) \varphi^{\prime}-\mu \frac{\Phi(\exp (-\varphi))-\exp (-\varphi)}{\exp (-\varphi)}\right\rangle d s
\end{gathered}
$$

is a $P_{\nu}$-martingale. Equating (4) with (5) leads to (3), that gives the second assertion of the Theorem.

\section{Weak convergence and limit of the system of particles}

Now, we show that the empirical process $\left\{\eta_{t}\right\}$ when it is renormalized, converges to a measure-valued process which is an extension of the Dawson-Watanabe superprocess [10].

The idea to pass to the limit consists in applying the Feller rescaling [12] : the number of particles, their mass and the branching rate are rescaled by considering that there are a very large number $N$ of particles, each of mass $\frac{1}{N}$ and of lifetime $\frac{1}{N \mu}$. 
Let $\left\{Y^{(n)}\right\}_{n>1}$ be the sequence of rescaled processes and consider at the $n^{\text {th }}$ stage an initial measure consisting of $N_{n}$ cells each of whom is assigned a mass $\frac{1}{N_{n}}$ and has an independent exponential lifetime of parameter $\mu_{n}=N_{n} \mu$, during which it moves according equation (1). At the end of its lifetime, it dies and leaves behind, at the location where it died, a random number of offspring ( 0 or 2 ) determined by the generating function $\Phi$. Suppose that the initial condition $\left\{Y_{0}^{(n)}\right\}_{n \geq 1}$ is convergent.

We prove the following convergence result :

Theorem 2 If $N_{n} \longrightarrow+\infty$ as $n \longrightarrow+\infty$, the sequence of rescaled processes $\left\{Y^{(n)}\right\}_{n>1}$ converges weakly (in distribution) in the space $D\left(\left[0,+\infty\left[, M_{F}(\mathbb{R})\right)\right.\right.$ to a measure-valued continuous process $\left\{Y_{t}, t \geq 0\right\}$, whose distribution $P$ satisfies the following martingale problem:

$\forall \varphi \in C_{b}^{2}(\mathbb{R})$

$$
M_{t}(\varphi) \triangleq\left\langle Y_{t}, \varphi\right\rangle-\left\langle Y_{0}, \varphi\right\rangle-\int_{0}^{t}\left\langle Y_{s}, D \varphi^{\prime \prime}+\left(F_{a} * \eta_{s}\right) \varphi^{\prime}\right\rangle d s
$$

is a P-martingale with the quadratic variation

$$
\langle M(\varphi)\rangle_{t}=\mu \int_{0}^{t}\left\langle Y_{s}, \varphi^{2}\right\rangle d s
$$

Proof. To prove weak convergence for the sequence of rescaled processes $\left\{Y^{(n)}\right\}_{n \geq 1}$ in $D\left(\left[0,+\infty\left[, M_{F}(\mathbb{R})\right)\right.\right.$, we may show tightness of $\left\{Y^{(n)}\right\}_{n \geq 1}$ in $D\left(\left[0,+\infty\left[, \dot{M}_{F}(\mathbb{R})\right)\right.\right.$ and then uniqueness of its limit points.

Part 1 : Tightness.

By Theorem 1.18 in ([10], page 11), we have just to show that the sequence $\left\{\left\langle Y^{(n)}, \varphi\right\rangle\right\}_{n \geq 1}$ is tight in $D\left(\left[0,+\infty[, \mathbb{R})\right.\right.$ for any function $\varphi$ in a dense subset of $C_{b}^{+}(\mathbb{R})$, provided that the following compact containment condition holds :

$\forall \epsilon>0, \forall T>0, \exists$ a compact set $\Gamma_{\epsilon, T} \subset M_{F}(\mathbb{R})$ such that :

$$
\inf _{n} P\left[Y_{t}^{(n)} \in \Gamma_{\epsilon, T} \quad \text { for } 0 \leq t \leq T\right] \geq 1-\epsilon .
$$

We show that the latter is satisfied when we replace $\mathbb{R}$ by its compactification $\overline{\mathbb{R}}=$ $\mathbb{R} \cup\{\infty\}$.

The compact containment of $\left\{Y^{(n)}\right\}_{n \geq 1}$ in $M_{F}(\mathbb{R})$ follows from the compact containment of $\left\{\left\langle Y^{(n)}, 1\right\rangle\right\}_{n \geq 1}$ in $\mathbb{R}$. Indeed, as $\left\langle Y_{t}^{(n)}, 1\right\rangle$ is a martingale for each $n$, we have :

$$
P\left[\sup _{0 \leq t \leq T}\left\langle Y_{t}^{(n)}, 1\right\rangle>K\right] \leq \frac{1}{K} E\left\langle 1, Y_{0}^{(n)}\right\rangle
$$


Since the initial measures $\left(Y_{0}^{(n)}\right)$ are convergent, $\frac{1}{K} E\left\langle 1, Y_{0}^{(n)}\right\rangle$ tends to 0 uniformly in $n$ as $K \longrightarrow \infty$. This implies that for each fixed $T>0$, given $\epsilon>0, \exists K>0$ such that

$$
P\left[\sup _{0 \leq t \leq T}\left\langle Y_{t}^{(n)}, 1\right\rangle \leq K\right] \geq 1-\epsilon
$$

We are only concerned with bounded $\varphi$, so the tightness of $\left\langle Y_{t}^{(n)}, \varphi\right\rangle$ for fixed $t$ follows a fortiori.

Now, we use the Aldous-Rebolledo criterion to show the tightness of $\left\{\left\langle Y^{(n)}, \varphi\right\rangle\right\}_{n}$ in $D([0,+\infty[, \mathbb{R})$ (see Theorem 1.17 in [10]).

For each $n \in N$, we consider a positive stopping time $\tau_{n}$ bounded by some finite constant $T$ and let $\varepsilon>0$. We must check that for any sequence $\left(\delta_{n}\right)$ such that $\delta_{n} \rightarrow 0$ as $n \rightarrow \infty$,

$$
\lim _{n \longrightarrow \infty} E_{n}\left(\int_{\tau_{n}}^{\tau_{n}+\delta_{n}}\left|\left\langle Y_{s}^{(n)}, D \varphi^{\prime \prime}+\frac{1}{N_{n}}\left(F_{a} * Y_{s}^{(n)}\right) \varphi^{\prime}\right\rangle\right| d s\right)=0
$$

and

$$
\lim _{n \rightarrow \infty} E_{n}\left(\int_{\tau_{n}}^{\tau_{n}+\delta_{n}}\left|\left\langle Y_{s}^{(n)}, 2 D\left(\varphi^{\prime}\right)^{2}+2 \mu \frac{\Phi(\exp (-\varphi))-\exp (-\varphi)}{\exp (-\varphi)}\right\rangle\right| d s\right)=0 .
$$

Thanks to the Strong Markov property satisfied by the process $\left\{Y_{s}^{(n)}\right\}$, We show that :

$$
\begin{gathered}
E_{n}\left(\int_{\tau_{n}}^{\tau_{n}+\delta_{n}}\left|\left\langle Y_{s}^{(n)}, D \varphi^{\prime \prime}+\frac{1}{N_{n}}\left(F_{a} * Y_{s}^{(n)}\right) \varphi^{\prime}\right\rangle\right| d s\right) \\
\leq \delta_{n}\left(K_{1} E_{n}\left\langle Y_{\tau_{n}}^{(n)}, 1\right\rangle+K_{2} E_{n}\left\langle Y_{\tau_{n}}^{(n)}, 1\right\rangle^{2}\right)
\end{gathered}
$$

where $K_{1}$ and $K_{2}$ are real constants independent of $n$. On another hand, we can see that :

$$
\left\langle Y_{t}^{(n)}, 1\right\rangle=\left\langle Y_{0}^{(n)}, 1\right\rangle+M_{t}^{n}(1)
$$

where $M_{t}^{n}(1)$ is a martingale with quadratic variation

$$
\left\langle M^{n}(1)\right\rangle_{t}=\int_{0}^{t}\left\langle Y_{s}^{(n)}, 2 \mu \frac{\Phi(\exp (-1))-\exp (-1)}{\exp (-1)}\right\rangle d s .
$$

Hence

$$
E_{n}\left\langle Y_{\tau_{n}}^{(n)}, 1\right\rangle=E_{n}\left\langle Y_{0}^{(n)}, 1\right\rangle
$$

Using Itô's formula, we have :

$$
E_{n}\left\langle Y_{\tau_{n}}^{(n)}, 1\right\rangle^{2} \leq E_{n}\left\langle Y_{0}^{(n)}, 1\right\rangle^{2}+K_{3} E_{n}\left\langle Y_{0}^{(n)}, 1\right\rangle
$$


where $K_{3}$ is a real constant independent of $n$. Consequently, if $\delta_{n} \rightarrow 0$ as $n \rightarrow 0$, then we obtain (8). The same techniques of calculation show that $(9)$ holds.

The tightness of the sequence $\left\{Y^{(n)}\right\}_{n \geq 1}$ in $D\left(\left[0,+\infty\left[, M_{F}(\overline{\mathbb{R}})\right)\right.\right.$ is proved.

Using Prohorov theorem, we conclude that the sequence $\left\{Y^{(n)}\right\}_{n \geq 1}$ is relatively compact in the weak topology on $M_{F}(\overline{\mathbb{R}})$.

Part 2 : Identification of limit points of the sequence $\left\{Y^{(n)}\right\}_{n \geq 1}$.

We consider a sequence of test functions of the form $f_{n}=1-\frac{\varphi}{N_{n}}$ with $\varphi \geq 0, \varphi \in$ $C_{b}^{2}(\overline{\mathbb{R}})$ and apply the martingale characterization to $N_{n} Y^{(n)}$. Using Taylor's theorem, the expression for the martingale becomes :

$$
\begin{aligned}
& \exp \left\langle Y_{t}^{(n)}, N_{n} \log \left(1-\frac{\varphi}{N_{n}}\right)\right\rangle-\exp \left\langle Y_{0}^{(n)}, N_{n} \log \left(1-\frac{\varphi}{N_{n}}\right)\right\rangle \\
& -\int_{t}^{t+u}\left\langle Y_{s}^{(n)}, \frac{-D \varphi^{\prime \prime}-\left(F_{a} * Y_{s}^{(n)}\right) \varphi^{\prime}}{1-\varphi / N_{n}}+\frac{\frac{1}{2} \mu \varphi^{2}}{1-\varphi / N_{n}}\right\rangle \exp \left\langle Y_{s}^{(n)}, N_{n} \log \left(1-\frac{\varphi}{N_{n}}\right)\right\rangle d s
\end{aligned}
$$

is a $P^{(n)}$-martingale. Letting $N_{n} \longrightarrow+\infty$ when $n$ tends to infinity, (10) gives that for any limit point $Y$. of $Y^{(n)}$ :

$$
\exp \left(-\left\langle Y_{t}, \varphi\right\rangle\right)-\exp \left(-\left\langle Y_{0}, \varphi\right\rangle\right)-\int_{0}^{t}\left\langle Y_{s},-D \varphi^{\prime \prime}-\left(F_{a} * Y_{s}\right) \varphi^{\prime}+\mu \frac{\varphi^{2}}{2}\right\rangle d s
$$

should be a martingale.

To justify this conclusion, we use Theorem 8.10 in ([11], page 234). We may show that : $\forall 0 \leq t_{1} \leq \ldots \leq t_{k} \leq t<t+u, \quad h_{1}, \ldots, h_{k} \in C_{b}\left(M_{F}(\overline{\mathbb{R}})\right), \varphi \geq 0$ and $\varphi \in C_{b}^{2}(\overline{\mathbb{R}})$

$$
\begin{aligned}
& \lim _{n \longrightarrow \infty} E\left[\left(\exp \left(-\left\langle Y_{t+u}^{(n)}, \varphi\right\rangle\right)\right.\right.-\exp \left(-\left\langle Y_{t}^{(n)}, \varphi\right\rangle\right)-\int_{t}^{t+u}\left\langle Y_{s}^{(n)},-D \varphi^{\prime \prime}-\left(F_{a} * Y_{s}^{(n)}\right) \varphi^{\prime}\right. \\
&\left.\left.\left.+\mu \frac{\varphi^{2}}{2}\right\rangle\right) \prod_{i=1}^{k} h_{i}\left(Y_{t_{i}}^{(n)}\right)\right]=0 .
\end{aligned}
$$

Since all the $h_{i}$ are bounded and using the calculation above to apply the Aldous-Rebolledo criterion, we can easily obtain (11) by the Dominated Convergence Theorem.

It follows that a limit point process $\left\{Y_{t}, t \geq 0\right\}$ satisfies :

$\forall \varphi \geq 0, \varphi \in C_{b}^{2}(\overline{\mathbb{R}}),\left\langle Y_{t}, \varphi\right\rangle$ is a semimartingale and

$$
\begin{aligned}
& M_{t}(\varphi) \triangleq\left\langle Y_{t}, \varphi\right\rangle-\left\langle Y_{0}, \varphi\right\rangle-\int_{0}^{t}\left\langle Y_{s}, D \varphi^{\prime \prime}+\left(F_{a} * Y_{s}\right) \varphi^{\prime}\right\rangle d s \\
& \text { is a martingale with }\langle M(\varphi)\rangle_{t}=\mu \int_{0}^{t}\left\langle Y_{s}, \varphi^{2}\right\rangle d s .
\end{aligned}
$$

Part 3 : Uniqueness of the limit points.

To prove that the solution to this martingale problem is unique, we appeal to Theorem 
V.5.1 in ([25], page 310) based on the techniques in ( [7], page 201 and [20]). So we only have to show that the function $F_{a} * \mu$ is Lipschitz in the sense of Perkins [25], that is, if :

$$
L I P(\mathbb{R})=\left\{\varphi: \mathbb{R} \rightarrow \mathbb{R}:\|\varphi\|_{\infty} \leq 1 \text { and }|\varphi(x)-\varphi(y)| \leq|x-y|, \forall x, y \in \mathbb{R}\right\}
$$

and $d$ denotes the Wasserstein metric on $M_{F}(\mathbb{R})$ :

$$
d(\mu, \nu)=\sup \{|\langle\mu, \varphi\rangle-\langle\nu, \varphi\rangle|: \varphi \in L I P(\mathbb{R})\},
$$

we have to show that there exists a non-decreasing function $L: \mathbb{R}^{+} \longrightarrow \mathbb{R}^{+}$such that :

$$
\left|\left(F_{a} * \mu\right)(x)-\left(F_{a} * \nu\right)(y)\right| \leq L(\mu(1) \vee \nu(1))[d(\mu, \nu)+|x-y|], \forall x, y \in \mathbb{R} .
$$

Let $x, x^{\prime} \in \mathbb{R}$ et $\mu, \mu^{\prime} \in M_{F}(\mathbb{R})$, we have that

$$
\begin{aligned}
\left|\left(F_{a} * \mu\right)(x)-\left(F_{a} * \mu^{\prime}\right)\left(x^{\prime}\right)\right| & \leq\left|\int_{\mathbb{R}} F_{a}(x-y) \mu(d y)-\int_{\mathbb{R}} F_{a}(x-y) \mu^{\prime}(d y)\right| \\
& +\left|\int_{\mathbb{R}} F_{a}(x-y) \mu^{\prime}(d y)-\int_{\mathbb{R}} F_{a}\left(x^{\prime}-y\right) \mu^{\prime}(d y)\right|
\end{aligned}
$$

Let us choose $K_{1}$ large so that $\left\|\frac{F_{a}}{K_{1}}\right\|_{\infty} \leq 1$. Since $F_{a}$ is bounded and Lipschitz continuous in $\mathbb{R}$ (see [8]), then $\frac{F_{a}}{K_{1}} \in \operatorname{LIP}(\mathbb{R})$.

We obtain :

$$
\left|\left(F_{a} * \mu\right)(x)-\left(F_{a} * \mu^{\prime}\right)\left(x^{\prime}\right)\right| \leq K\left[1+\mu(1) \vee \mu^{\prime}(1)\right]\left(d\left(\mu, \mu^{\prime}\right)+\left|x-x^{\prime}\right|\right)
$$

where $K$ is a constant. We take $L(r)=K(1+r)$, that completes the proof of uniqueness for the martingale problem (6).

Hence, the sequence of rescaled processes $\left\{Y_{t}^{(n)}, t \geq 0\right\}_{n \geq 1}$ converges weakly to the process $\left\{Y_{t}, t \geq 0\right\}$ which is the unique solution to the martingale problem (6).

Since we have worked in the space $M_{F}(\overline{\mathbb{R}})$ to guarantee the compact containment condition, we have to check that the limit of the sequence of processes $\left\{Y^{(n)}\right\}_{n \geq 1}$ takes its values in the space $M_{F}(\mathbb{R})$, that is no mass escaped to infinity when we passed to the limit. This is done by using a similar technique to that in [22].

From (6) and (7), we recognize that $\left\{Y_{t}, t \geq 0\right\}$ is a generalization of the DawsonWatanabe superprocess.

\section{From a martingale characterization to a stochastic partial differential equation}

Our first result in this direction : 
Theorem 3 The process $\left\{Y_{t}, t \geq 0\right\}$ is solution of the non linear stochastic differential equation in a space of measures :

$$
d Y_{t}=D \frac{d^{2}}{d x^{2}} Y_{t} d t-\frac{d}{d x}\left[Y_{t}\left(F_{a} * Y_{t}\right)\right] d t+d M_{t},
$$

where $M_{t}$ is a continuous martingale measure (in the sense of [28]), with covariance measure $\mu Y_{s}(d x) d s$.

Proof. The proof of this theorem is based on the arguments used in [22]. We would like to represent the martingale term $M_{t}(\varphi)$ in (6) as a stochastic integral with respect to a white noise. We use the extension result in ([23], Proposition 2.6), and extend the map $\varphi \longrightarrow M_{\text {. }}(\varphi)$ from $C_{b}^{2}(\mathbb{R})$ to $L^{2}(\gamma)$, where $\gamma$ is the measure on $\mathbb{R}$ defined by :

$$
\gamma(d x)=E\left[\int_{0}^{T} \mu Y_{s}(d x) d s\right] .
$$

This defines a continuous finite orthogonal martingale measure. Hence, there exists a random predictible real-valued measure $I$ on $\mathcal{B}([0, T]) \otimes \mathcal{F}$, defined by $\langle M(A)\rangle_{t}=$ $\int_{0}^{t} \int_{\mathbb{A}} I(d x, d s)$ P-p.s, $\forall t>0$. Moreover, we can construct a stochastic integral with respect to $M$, for all function $f$ such that : $E\left[\int_{0}^{t} \int_{\mathbb{A}} f^{2}(s,, x) I(d x, d s)\right]<\infty$. This stochastic integral is denoted by $\int_{0}^{t} \int_{\mathbb{A}} f(s, x) M(d x, d s)$.

Heuristically, if we suppose that for every $t>0, Y_{t}$ is absolutely continuous with respect to the Lebesgue measure on $\mathbb{R}$, then the density $f(t, x)$ of $Y_{t}$ will satisfy :

Theorem 4 The density $f(t, x)$ of $Y_{t}$, with respect to the Lebesgue measure, is a weak solution to the following SPDE:

$$
\frac{\partial f(t, x)}{\partial t}=D \frac{\partial^{2} f(t, x)}{\partial x^{2}}-\frac{\partial}{\partial x}\left[f(t, x)\left(F_{a} * f(t, .)\right)(x)\right]+\sqrt{\mu f(t, x)} \dot{W}(t, x),
$$

with $\dot{W}$ defined by :

$$
W(d t, d x)=\dot{W}(t, x) d t d x,
$$

where $W(d t, d x)$ is a Gaussian white noise (in the sense of [28]).

Proof. Using that $Y_{t}(d x)=f(t, x) d x$, we should like to write :

$$
M_{t}(\varphi)=\int_{0}^{t} \int_{\mathbb{R}} \sqrt{\mu f(t, x)} \varphi(x) W(d x, d s)
$$


where $W(d x, d s)$ is a Gaussian white noise.

To $M_{t}(\varphi)$, there corresponds an orthogonal martingale measure $M(d x, d s)$ in the sense of ([28], chap 2) whose quadratic variation measure is

$$
\langle M\rangle(d s d x)=f(s, x) d s d x .
$$

We can construct the white noise as follows :

$$
\begin{array}{r}
W_{t}(\varphi)=\int_{0}^{t} \int_{\mathbb{R}} \frac{1}{\sqrt{\mu f(t, x)}} 1_{\{f(t, x) \neq 0\}} \varphi(x) M(d x, d s) \\
\quad+\int_{0}^{t} \int_{\mathbb{R}} 1_{\{f(t, x)=0\}} \varphi(x) \widetilde{W}(d x, d s)
\end{array}
$$

with $\widetilde{W}$ a white noise independent of $Y_{t}(d x)$.

Then, $W_{t}$ is a cylindrical Brownian motion that extends to a Gaussian white noise $W(d x, d s)$. Substituting in $(6)$, we get :

$$
\begin{aligned}
& \int_{\mathbb{R}} \varphi(x) f(t, x) d x=\int_{\mathbb{R}} \varphi(x) f(0, x) d x+D \int_{0}^{t} \int_{\mathbb{R}} \varphi^{\prime \prime}(x) f(t, x) d x d s \\
& +\int_{0}^{t} \int_{R}\left(F_{a} * f(s, .)\right)(x) \varphi^{\prime}(x) f(s, x) d x d s+\int_{0}^{t} \int_{R} \sqrt{\mu f(t, x)} \varphi(x) W(d x, d s) .
\end{aligned}
$$

We recognize that (14) is a weak version of the nonlinear stochastic partial differential equation (12).

\section{Concluding remarks}

A solution to (12) exhibits a kind of clustering phenomena : on one hand, the convolution form in the advective term of (12) expresses the attraction of cells to higher density, a mechanism that leads to aggregation formation, and on the other hand, the last term in (12) represents the stochastic imbalence between births and deaths in space and time. This term occurs because birth is always next to a living cell, while death can occur anywhere. The accumulation of these small scale density fluctuations develops patches and voids on large scales. So, aggregation here is a consequence of both the nonlinear attractions between cells due to the chemosensory behavior and the branching mechanism. These ideas are still new for biologists and oceanographers.

To our knowledge, a stochastic partial differential equation of form (12) is unknown in both biological and mathematical literatures. In [1], the authors have investigated the mathematical analysis (existence, uniqueness and positivity of solutions) for the model described by (12) without the stochastic term (that is the branching phenomenon has 
been ignored in the phytoplankton story). In addition, existence of nonuniform stationary solutions has been established. This asymptotic result confirms that nonlinear interactions between phytoplankton cells at small scales can produce aggregations at large scales. We intend, in a future work, to explore the asymptotic behavior of the stochastic equation (12).

Finally, we would like to point out that in [9], the authors have conceived and simulated a numerical Individual-Based Model (IBM) showing the behavior of the system of stochastic differential equations in (1). All the simulation results show formation of clusters, qualitatively by visualizing aggregates formation and quantitatively by using an aggregation indicator, confirming that our mathematical model is really an aggregation model.

\section{Bibliographie}

[1] M. Adioui, O. ARino AND N. El SAADI, «A nonlocal model of phytoplankton aggregation. », Nonlinear Analysis : Real World Applications 6 (2005) $593-607$.

[2] A. L. Alldredge AND M. Silver, « Characteristics, dynamics and significance of marine snow. », Progress in oceanography 20 (1983) 41 - 82 (Springer-Verlag, New York, 119).

[3] A. L. Alldredge, C. Gotschalk, U. Passow and U. Riebesell, « Mass aggregation of diatom blooms : Insights from a mesocosm study. », Deep-sea Research II 421 (1995) $9-27$.

[4] W. Bell AND R. Mitchell, « Chemotactic and growth responses of marine bacteria to algal extracellular products. », Biol. Bull. 143 (1972) $265-277$.

[5] H. C. Berg And E. M. PurCell, «Physics of chemoreception. », Biophys. J. 20 (1977) $193-219$.

[6] H. G. Dam And D. T. Drapeau, « Coagulation efficiency, organic-matter glues and the dynamics of particles during a phytoplankton bloom in a mesocosm study. », Deep-sea Research II 421 (1995) $111-123$.

[7] P. Donnelly and T. G. Kurtz, « Particle representations for measure-valued population models. », The Annals of Probability 27 (1) (1999) $166-205$.

[8] N. EL SAADI, «Modélisation et études mathématique et informatique de populations structurées par des variables aléatoires. Application à l'agrégation du phytoplancton. », Thèse de Doctorat, Université de Pau et des pays de l'Adour (2004).

[9] N. El SAADI AND A. BAH, « An individual-based model for studying the aggregation behavior in phytoplankton. ", (in revision).

[10] A. M. ETHERIDGE, « An introduction to superprocesses. », University Lecture series 20. American Mathematical society (2000).

[11] S. N. Ethier And T. G. KuRTZ, « Markov Processes : Characterization and Convergence. », Wiley, New York (1986). 
[12] W. FELLER, « Diffusion processes in genetics. », Proc Second Berkeley Symp (1951) 227 246.

[13] W. K. FITT, « Chemosensory responses of the symbiotic dinoflagellate Symbiodinium microadriatica (Dinophycae). », J. Phycol 21 (1985) $62-67$.

[14] S. W. Fowler AND G. A. KNAUER, « Role of large particles in the transport of elements and organic compounds through the ocean water column. », Prog. Oceanogr 16 (1986) $147-194$.

[15] P. Gros, « La représentation de l'espace dans les modèles de dynamique des populations. », IFREMER. Centre de Brest (2001).

[16] G. A. JACKSON, « Simulating chemosensory responses of marine microorganisms. », Limnol.Oceanogr 32 (6) (1987) $1253-1266$.

[17] G. A. JACKSON, « Simulation of bacterial attraction and adhesion to falling particles in an aquatic environment. », Limnol.Oceanogr 34 (3) (1989) $514-530$.

[18] T. KIORBOE, «Formation and fate of marine snow : small-scale processes with large-scale implications. », Sci. Mar 655 (Suppl.2) (2001) $57-71$.

[19] T. Kiorboe, C. Lundsgaard, M. olesen and J. L. S. Hansen, « Aggregation and sedimentation processes during a spring phytoplankton bloom : A field experiment to test coagulation. », Journal of Marine Research 52 (1994) 297 - 323.

[20] T. G. KURTZ, « Martingale problems for conditional distributions of Markov processes. », Elect. J. Prob 3 (1998), paper 9.

[21] T. H. Mague, E. Friberg, D. J. Hughes and I. Morris, « Extracellular release of carbon by marine phytoplankton : a physiological approach. », Limnol. Oceanogr 25 (1980) $262-279$.

[22] S. MElEard AND S. RoElly, « Interacting measure branching processes. Some bounds for the support. ", Stochastics and Stochastics Reports 44 (1993) $103-121$.

[23] S. Meleard and S. Roelly, « Discontinuous measure-valued branching processes and generalized stochastic equations. », Math. Nachr. 154 (1991) 141 - 156.

[24] D. Morale, V. Capasso and K. Oelschlager, «An interacting particle system modelling aggregation behavior : from individuals to populations. », J. Math. Biology, 50 (1) (2005) $49-66$.

[25] E. PERKINS, « Dawson-Watanabe superprocesses and measure-valued diffusions. », Ecole d'été de probabilités de Saint-Flour (1999). Lecture notes in mathematics 1781. SpringerVerlag (2002).

[26] H. J. Spero And M. MoréE, « Phagotrophic feeding and its importance to the life cycle of the holozoic dinoflagellate, Gymnodinium fungiforme. », J. Phycol 17 (1981) $43-51$.

[27] H. J. SPERo, « Chemosensory capabilities in the phagotrophic dinoflagellate Gymnodinium Fungiforme. », J. Phycol 21 (1985) $181-184$.

[28] J. B.WALSH, «An introduction to stochastic partial differential equations. », Ecole d'été de probabilités de Saint Flour 1180. Springer-Verlag (1986). 